\title{
Modelo Explicativo del Rendimiento Académico Asociado a Estilos de Crianza, Agresión y Resentimiento en Adolescentes Peruanos
}

\author{
Explanatory Model of Academic Performance Associated with Parenting Styles, \\ Aggression and Resentment in Peruvian Adolescents
}

\author{
Jesús Dámaso-Flores ${ }^{1}$ y Antonio Serpa-Barrientos ${ }^{2}$
}

\begin{abstract}
Resumen
El estudio tuvo como objetivo, primero establecer un modelo explicativo estructural del rendimiento académico en función a estilos de crianza, conducta agresiva y resentimiento como variables mediadoras en adolescentes escolares; y segundo evidenciar las bondades psicométricas de los instrumentos utilizados desde el Modelo Estructural. La muestra estuvo conformada por 766 estudiantes peruanos de educación secundaria con edades de 11 a 18 años. Las evidencias de confiabilidad de los instrumentos se obtuvieron a través del coeficiente alfa y omega con valores aceptables. El análisis desde el modelo estructural, cumpliendo los supuestos de normalidad multivariado y la no colinealidad evidencian el ajuste del modelo explicativo del rendimiento académico asociado a las variables estilos de crianza, conducta agresiva y resentimiento. Se encontró un efecto directo acentuado del resentimiento frente a la variable de respuesta (rendimiento académico). Los estilos de crianza autonomía psicológica y control conductual aportan en los niveles de agresividad y resentimiento.
\end{abstract}

Palabras clave: rendimiento académico, estilos de crianza, conducta agresiva, resentimiento

\begin{abstract}
The objective of the study was, first, to establish a structural explanatory model of academic performance based on parenting styles, aggressive behavior and resentment as mediating variables in school adolescents; and second, to show the psychometric benefits of the instruments used from the Structural Model. The sample consisted of 766 Peruvian high school students aged 11 to 18 years. The evidence of reliability of the instruments was obtained through the alpha and omega coefficients with acceptable values. The analysis from the structural model, fulfilling the assumptions of multivariate normality and non-collinearity, shows the adjustment of the explanatory model of academic performance associated with the variables of parenting styles, aggressive behavior and resentment. An accentuated direct effect of resentment was found against the response variable (academic performance). The styles of parenting, psychological autonomy and behavioral control contribute to the levels of aggressiveness and resentment.
\end{abstract}

Keywords: academic performance, parenting styles, aggressive behavior, secondary education, resentment

\footnotetext{
${ }^{1}$ Docente de la Facultad de Ciencias de la Salud, Escuela de Psicología, Universidad César Vallejo. Miembro del equipo técnico de capacitación y evaluación de programas comunitarios. Dirección de seguridad ciudadana de la Policia Nacional del Perú. Perú. Correo: jdamasof@ucvvitual.edu.pe

${ }_{2}^{2}$ Docente de la Universidad Nacional Mayor de San Marcos. Auditor Líder de Sistema de Gestión de la Calidad, Normas ISO 9001. Correo: aserpab@unmsm.edu.pe

Revista Iberoamericana de Diagnóstico y Evaluación - e Avaliação Psicológica. RIDEP · Nº2 · Vol.1 · 5-15 · 2022

ISSN: 1135-3848 print /2183-6051online
} 


\section{Introducción}

El rendimiento académico en la etapa de la adolescencia, no solo está referido a la convergencia de habilidades y destrezas tanto de los estudiantes como de los profesores, sino también de las diversas dificultades, propias de esta etapa, que enfrenta el adolescente $y$ probablemente van a trascender en su desarrollo y proceso educativo. En este marco, por ejemplo, muchas instituciones educativas denotan dificultades relacionados al nivel de conocimientos obtenidos por los estudiantes en alguna área específica, lo cual generalmente es atribuido no solamente al desempeño individual sino también al entorno.

Desde esta perspectiva del ámbito familiar y de los mismos estudiantes, los logros en el aprendizaje pueden tomar importancia en razón de que se visualizan actitudes y conductas que pueden ser favorables o desfavorables para las actividades escolares y sus logros. En este sentido, Leiva et al. (2019), en un estudio sobre dificultades emocionales y conductuales en la escuela, reportan que de acuerdo a las correlaciones encontradas entre las puntuaciones otorgadas por profesores, padres y cuidadores, las dificultades presentadas en el contexto escolar y en el hogar, muestran una cierta estabilidad en el sentido que aquellos niños que tienen dificultades para adaptarse al medio escolar tendrían una menor probabilidad de ajustarse otros medios sociales o viceversa, lo que en términos de respuestas escolares podría reflejarse en dificultades para aprender o para ajustarse a las normas de conducta que rigen el aula.

Tomando estos hallazgos como referencia se puede relacionar los logros escolares con aspectos personales y sociales que involucra actitudes y comportamientos que están presentes en el contexto familiar. Al respecto, algunos autores señalan que los factores sociales tienen una gama mucho más amplia que abarca el entorno familiar, cultura, medios de comunicación, etc.; y dentro del entorno familiar se derivan el nivel socioeconómico, estilos parentales y estructura familiar (Enríquez et al., 2013)

Por su parte, Quintero (2017), refiere que puede decirse que el rendimiento académico viene condicionado por el equilibrio afectivo y emocional del estudiante, ya que la familia es fundamental en la formación del individuo, la acumulación de experiencias adquiridas y las actitudes desarrolladas servirán significativamente para modelar la personalidad y fortalecer la instrucción académica, a su vez por las relaciones que mantiene con los miembros de su familia.

Otros estudios reportaron también que la familia y su dinámica forman parte de los factores que van a intervenir en el desempeño académico (Anabalón et al., 2008; Baeza, 2000; Rodriguez \& Guzmán, 2019), que los antecedentes familiares son el determinante individual de mayor importancia y peso para el rendimiento académico alcanzado y en términos generales, el aprendiz suele lograr el éxito académico cuando sus padres le ofrecen apoyo y orientación (Adell, 2002; Craig, 2001), que entre los factores influyentes en el desempeño académico de los alumnos, resaltan los estilos de crianza, ya que estos se relacionan con la autorregulación y la motivación, así como con el desarrollo de estrategias de aprendizaje (Lozano, 2003; Vallejo \& Mazadiego, 2006; Salazar et al., 2009; Jiménez, 2009; Domínguez, 2012), que las circunstancias ejercen un efecto moldeador sobre las ideas de los padres y, a su vez influyen en la configuración del estilo educativo familiar (Ramírez, 2005), que los padres generalmente ejercen una fuerte influencia a través de la educación que proporcionan a sus hijos en los hogares, situación que también afecta al desempeño académico (González-Pienda \& Núñez, 2005), que los padres son los principales gestores para el desarrollo de los hijos y son los pioneros en practicar los estilos de crianza, que influyen en los procesos de socialización (Izzedin \& Pachajoa, 2009; Henríquez, 2014; Carrión, 2015).

Estos estudios mencionados pueden fundar que los determinantes del rendimiento escolar, entendido como el nivel de conocimientos o los logros obtenidos por el estudiante en un área específica, están relacionados, entre otros aspectos, con componentes de tipo familiar como los estilos de crianza, los cuales a su vez pueden estar matizados en alguna medida con cargas de agresión. Para Quintero (2017), las variables relativas al entorno familiar son consideradas el principal predictor del rendimiento académico, afirmando que los padres son los primeros 
educadores de las normas de conducta y comportamiento conforme a las reglas y principios aceptados para la convivencia humana y el desarrollo de su aprendizaje.

En cuanto a los estilos de crianza, constituyen una forma que tienen los padres de responder a las emociones de sus hijos o como una forma de relacionarse para elaborar sus funciones, recogen un conjunto articulado de pensamientos, sentimientos y actitudes que se desarrolla en torno a la crianza de los hijos y está sumido en el proceso de socialización e interacción social (Torio-López et al., 2013). Según este planteamiento las prácticas de crianza pueden llegar a ocupar un papel fundamental en la adquisición de valores y normas de convivencia, sin embargo, al debilitarse esta función de los estilos de crianza puede distorsionarse, proyectando una inadecuada socialización que pueden generar conductas reactivas o pueden convertirse en predictores de desajustes emocionales. Así, un adolescente que llegue a esta etapa y experimente sentimientos de ansiedad, de hostilidad, de rechazo, de trato injusto o de situaciones desfavorables de exclusión en el contexto familiar, especialmente por sus padres, pueden generar sentimientos de ira y venganza, elementos que configuran un resentimiento, que puede ser caracterizado básicamente por una conducta de hacer daño a otros. Al respecto Bausela (2018), refiere que la ansiedad es una variable que predice el bajo rendimiento académico en función de la presencia de otras variables predictoras, como la atribución causal del fracaso del escolar.

Para Darling y Steinberg (1993) el estilo de crianza es como una constelación de actitudes hacia los niños, que son comunicadas hacia él y que, tomadas en conjunto, crean un clima emocional en que se expresan las conductas de los padres. Los autores identifican tres componentes de los estilos de crianza: el control conductual, la responsividad parental o compromiso y la autonomía psicológica, los cuales se han examinado a través de una escala, desarrollada por Lawrence Steinberg. La subescala de Compromiso evalúa el grado en que el adolescente percibe conductas de acercamiento emocional, sensibilidad en interés provenientes de sus padres. La subescala de Control Conductual evalúa el grado en que el padre es percibido como controlador o supervisor del comportamiento del adolescente. Finalmente, la subescala Autonomía Psicológica evalúa el grado en que los padres emplean estrategias democráticas, no-coercitivas y animan a la individualidad y autonomía.

Resulta indiscutible que la influencia de los padres representa un componente muy importante en la educación de sus hijos, expresados en los estilos o prácticas de crianza, sin embargo, esta relación requiere un análisis de asociación con otras variables que también pueden influir en el rendimiento académico de manera directa e indirecta como la agresión y resentimiento, que suelen expresar los adolescentes en su interacción en los entornos escolares, dando lugar probablemente a estándares bajos en sus aprendizajes. Sobre el particular, el Programa para la Evaluación Internacional de Estudiantes (PISA) da a conocer que Perú es uno de los países de Latinoamérica que tiene los desempeños académicos más bajos de sus estudiantes. (Ministerio de Educación, 2017)

Respecto a la relación entre el rendimiento académico, la agresión y el resentimiento no se han encontrado estudios multivariados. La agresión es un tema que ha llamado la atención a muchos investigadores que lo han estudiado en relación a diversas variables, por ejemplo, con el rendimiento académico, encontrando relaciones inversas (Gil, 2015; Estévez \& Jiménez, 2015; Domínguez et al., 2017). También se ha estudiado con las relaciones intrafamiliares, encontrándose que esta variable es como un mecanismo explicativo de la conducta violenta en adolescentes escolarizados (Bonilla et al., 2017). En suma, la conducta de agresión se encuentra presente en los escenarios escolares coligado con otras variables concomitantes de la escuela.

Un estudio en la sociedad peruana sobre problemas sociales de mayor impacto, realizado por el Ministerio de Salud (2017) a través del Área de Epidemiologia reportó que el $73 \%$ de estudiantes de 12 a 17 años sufrieron violencia física en su colegio, teniendo como particularidad más notoria el hostigamiento (95\%). El Sistema Especializado en Reporte en Casos de Violencia Escolar (SISEVE) del Ministerio de Educación (2018) reportó un $76 \%$ de casos de violencia escolar a nivel nacional de los cuales $57 \%$ corresponden a ciudad capital de Lima. 
Sobre la agresión, clásicamente se explica como una conducta instintiva, impulsiva con la intención de dañar, sin embargo, más reciente y aceptado por la comunidad científica encontramos la postura de autores como Bandura (1973), quién destacó los aspectos sociales a través de experimentos para intentar explicar su naturaleza a través de la observación. De aquí se puede precisar que la conducta agresiva, no solo se aprende sino también se enseña. En esta línea encontramos el trabajo de Del Barrio et al. (2003) quienes reportaron que aquellos niños que ven televisión con contenidos violentos tienen una conducta más violenta en el futuro. Estas evidencias pueden denotar una latente agresión en los escolares. Para Buss y Perry (1992), la agresividad lo consideran como un aspecto individual, que se convierte en un hábito de acuerdo a la situación vivenciada por el individuo, si este se instaura como una reacción habitual, se puede hablar de un estilo de personalidad. A partir de este planteamiento construyen el denominado Aggression Questionnaire, un cuestionario para medir la agresión que puede ser configurado como una acción externa, abierta, objetiva y observable.

En cuanto al resentimiento, desde una perspectiva filosófica, se destaca como la imposibilidad de expresar activamente los sentimientos negativos y agresivos hacia otras personas (Nietzsche, 2011). Desde la perspectiva psicológica, Dorsch (1982) lo describe como el volver a experimentar un sentimiento doloroso frecuentemente inconsciente de envidia u odio. Para Gibu (2016), la formación del resentimiento parte de un impulso de venganza, por un deseo de desquitarse con alguien por un daño padecido o una postergación y como es parte de una personalidad, habituada a ocultar en su interior los sentimientos de rechazo y violencia. RosalesSarabia et al. (2018) hacen referencia que el papel de la ira persistente y los pensamientos negativos, son elementos centrales para el resentimiento, proponiendo que ocurre cuando una persona experimenta ira de manera persistente e intensa a consecuencia de una transgresión, ello genera un sesgo cognoscitivo negativo que promueve pensamientos más punitivos.

En el contexto peruano, León (1987), plantea al resentimiento como una reacción que implica un sentimiento de impotencia ante una injusticia o un agravio. En un intento de evaluar este constructo construyó una escala dicotómica. Instrumento que fue utilizado en algunas investigaciones relacionadas con otras variables como agresividad en adolescentes (Obregón, 2017), Agresión y Autoestima en adolescentes de conducta antisocial (Dámaso, 1994). Se notó inconsistencia en su operacionalizaciòn al ser sometido al método de análisis factorial. El presente estudio evaluó también la escala para darle mayor potencia como instrumento de investigación.

Respecto a las variables mencionadas, se han encontrado estudios de manera aislada que reportan hallazgos unilaterales que, si bien son importantes para el estudio de la persona como ser individual, se encuentran sesgos para un entendimiento integral. Por ello el estudio tuvo como propósito establecer un modelo explicativo del rendimiento académico en función a los estilos de crianza, la agresión y el resentimiento, así como también describir el resentimiento como una variable mediadora. Finalmente evidenciar las bondades psicométricas de los instrumentos utilizados desde el Modelo de Ecuaciones Estructurales (SEM).

\section{Método}

\section{Participantes}

Participaron 766 estudiantes, de los cuales 405 (53\%) fueron mujeres y 361 (47\%) varones, provenientes de instituciones educativas estatales, con edades que van desde 11 a 18 años $(M E=14.13, \quad D S=1.37)$. No se encontraron diferencias significativas al considerar el análisis según $\operatorname{sexo}\left(t_{(766)}=1.764, p=.078\right)$.

\section{Instrumentos}

Se utilizaron los siguientes: Escala de Estilos de Crianza (EEC; Steinberg, 1991), adaptado por Merino (2009) es un cuestionario de aplicación individual o colectiva que consta de 26 preguntas para adolescentes agrupada en tres dimensiones: Compromiso, Autonomía Psicológica y Control Conductual. Cuestionario de Agresión (CA; Buss \& Perry, 1992), adaptado por Matalinares (2012), es de aplicación individual o colectiva de 29 preguntas para adolescentes que integra cuatro 
dimensiones: agresividad física, agresividad verbal, ira y hostilidad. Y la Escala de Resentimiento (ER, León \& Romero, 1990), es un cuestionario dicotómico de aplicación individual y colectiva de 28 ítems para edades de 12 años en adelante. Para el rendimiento académico se utilizó los registros de calificaciones de cada uno de los estudiantes participantes del estudio.

\section{Estructura interna de los instrumentos}

Los tres instrumentos obtuvieron índices de ajuste global de $X^{2} / g l \leq 5$ (ver Tabla 1); asimismo, el índice de ajuste comparativo $C F I \geq .90, \mathrm{SRMR} \leq .08$ y RMSEA $\leq .05$, considerados los más adecuados. En la Escala de Resentimiento fue necesario reespecificar el modelo hasta obtener el ajuste más adecuado, retirando un total de 13 ítems $(2,4,7,9$, $13,14,17,19,20,24,25,26$ y 27), debido a que las cargas factoriales se encontraron por encima de la significancia $(p>.05)$ y cargas factoriales menores a .30 . Estos resultados permitieron confirmar evidencias de validez basado en la estructura interna de los instrumentos.

\section{Evidencias de confiabilidad de los instrumentos por el método de consistencia interna}

Las evidencias de la confiabilidad de la Escala de Estilos de Crianza (EEC), la Escala de Resentimiento (ER) y el Cuestionario de Agresión $(C A)$, se trabajaron a través del coeficiente alfa y Omega, obteniendo valores aceptables como: EEC $(\alpha=.78, \omega=.81)$, ER $(\alpha=.66, \omega=.66)$ y CA $(\alpha=.87$, $\omega=88)$, a excepción de la ER que se encuentran por debajo del valor ( $\alpha$ y $\omega \leq .70)$; Sin embargo, estos resultados a pesar de que se encuentran ligeramente por debajo del valor establecido como criterio permiten aceptar la fiabilidad del instrumento.

\section{Procedimiento}

Inicialmente se solicitó la autorización a la dirección de las instituciones educativas para la aplicación de los instrumentos y el asentimiento informado a los padres o tutores de cado uno de los estudiantes. Luego, los profesores y tutores comunicaron a los estudiantes los objetivos de la investigación y solicitaron su participación de manera voluntaria, cumpliendo de esta manera con el consentimiento informado.

Posteriormente se verificó la existencia de los puntajes extremos a través de la asimetría y curtosis en el rango [-2, 2] (Muthén \& Kaplan, 1985, 1992; Bandalos \& Finney, 2010), convenientemente con matrices: policórica (para ítems politómicos; EEC y CA) o tetracórica, (para ítems dicotómicos, ER), (Brown, 2006; Bandalos \& Finney, 2010, Ferrando $\&$ Anguiano-Carrasco, 2010). Con respecto a los supuestos de normalidad multivariado fue evaluado con el coeficiente (G2) de Mardia esperando magnitudes $<70$ (Rodríguez \& Ruiz, 2008).

Para brindar evidencias de validez basado en la estructura interna de los constructos, se procedió a realizar el análisis factorial confirmatorio a través del programa $\mathrm{R}$ Studio 3.0.1 bajo los siguientes criterios: el método de medias ponderadas de mínimos cuadrados y la estimación ajustada de la varianza (WLSMV) debido a que se aproximaron a una distribución multivariada y son datos ordinales (Gana \& Broc, 2019; Beaducel \& Herzberg, 2006); índices de ajuste como el SRMR $(\leq .05)$, RMSEA $(\leq .05)$, CFI $(\geq .95)$, y la proporción global $X^{2} / g l<2$ (Hu \& Bentler, 1999). Por último, para verificar las evidencias de la confiabilidad de los instrumentos se analizó a través del método de consistencia interna con los coeficientes $\alpha$ y $\omega \geq .70$.

Una vez verificado las propiedades psicométricas de cada uno de los instrumentos, se procedió a realizar el análisis del Path diagram haciendo uso del programa AMOS v.24, el cual nos permitió contrastar los objetivos, bajo los siguientes criterios: el ajuste del modelo estructural con la prueba global $X^{2} / g l$, índices de ajuste como RMSEA ( $\leq .05$ ), CFI ( $\geq .95$ ) (Hu \& Bentler, 1999) y los efectos directos e indirectos ( $\beta$ significativos, $p \leq .05$ ).

Finalmente se analizó la variable resentimiento como variable mediadora (Baron \& Kenny 1986) entre las variables: autonomía psicológica, control conductual y compromiso con el rendimiento académico, empleando el programa Jamovi 9.6, bajo los siguientes criterios: el efecto directo e indirecto es verificado como significativo $(p \leq .05)$. Y para ser considerado mediador en categoría parcial el efecto directo e indirecto son considerados significativos $(p \leq .05)$, y para una mediación de categoría total el efecto indirecto es considerado no significativo $(p \geq .05)$ (Ato \& Vallejo, 2015). 
Tabla 1. Índice de ajuste de los modelos de estructura interna de las variables: EEC, ER y CA

\begin{tabular}{|c|c|c|c|c|c|c|}
\hline Modelo estructural & $X^{2}$ & gl & $\mathrm{X}^{2} / \mathrm{gl}$ & CFI & SRMR & RMSEA \\
\hline EEC & 425.067 & 252 & 1.687 & .901 & .059 & .047 \\
\hline ER: M1 (28 ítems) & 443.649 & 350 & 1.267 & .734 & .061 & .029 \\
\hline M2 (15 ítems) & 109.554 & 90 & 1.127 & .942 & .050 & .027 \\
\hline $\mathrm{CA}$ & 427.763 & 257 & 1.664 & .940 & .051 & .046 \\
\hline
\end{tabular}

Nota. $\mathrm{X}^{2}$ : Chi cuadrado, gl: grados de libertad, CFI: índice de ajuste comparativo, SRMR: raíz residual estandarizada cuadrática media, RMSEA: error cuadrático medio de aproximación, EEC: escala de estilo de crianza, ER: resentimiento, CA: Conducta agresiva y M1: modelo sin re-especificación, M2: modelo re-especificado.

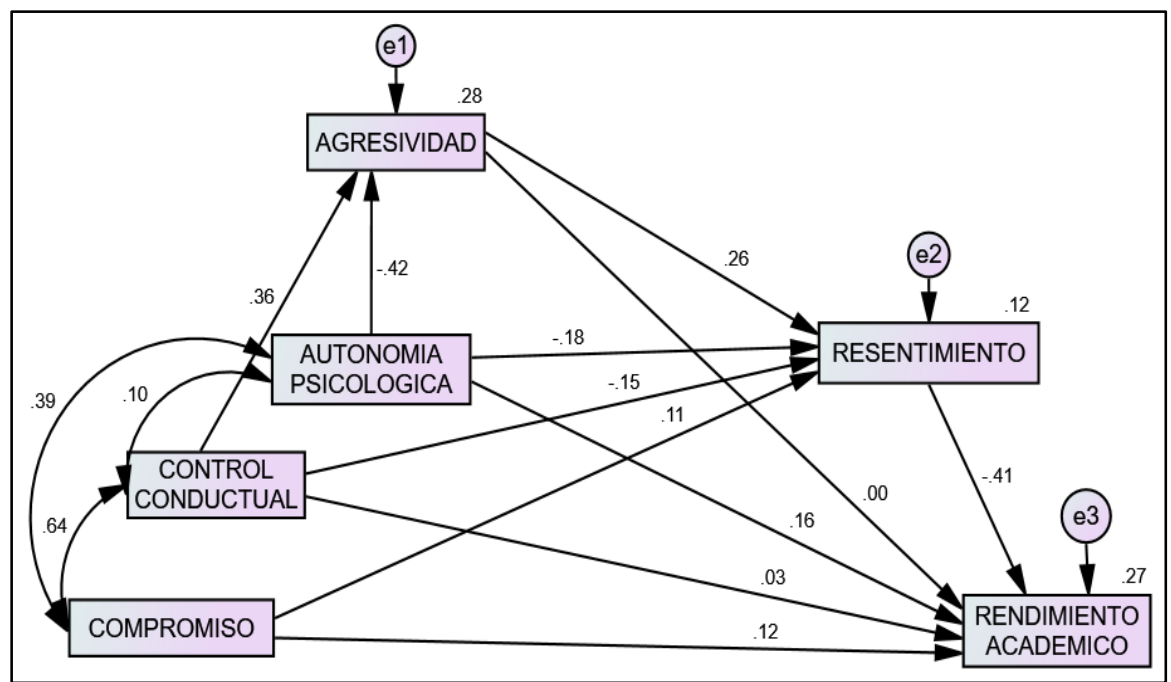

Figura 1. Modelo estructural de las variables de estudio

\section{Resultados}

\section{Descripción de puntajes extremos}

De acuerdo a los resultados del análisis descriptivo para los instrumentos EEC, ER y CA, éstos presentaron valores de asimetría y curtosis dentro del rango esperado [-2, 2], a excepción de los ítems 9 y 26 tanto en asimetría y curtosis de la escala resentimiento (ER). Con respecto al cumplimiento del supuesto de normalidad multivariado se obtuvo un coeficiente de Mardia $>70(\mathrm{G} 2=74.44, \mathrm{Z}=38.763, p<.00$; aproximándose a la distribución normal multivariada.

\section{Modelo estructural explicativo}

En cuanto a los índices de ajuste del modelo estructural explicativo se han obtenido resultados de $X^{2}=7.729$, con $g l=2$ y una proporción de $X^{2} / g l=3.865$; asimismo, CFI=.995, SRMR $=.029$ y RMSEA $=.061$, los cuales permitieron aceptar el modelo propuesto como unos de los objetivos principales para su explicación del rendimiento académico como variable de respuesta (Ato \& Vallejo, 2015). (ver Figura 1).

\section{Efecto directo}

Con respecto a la explicación de la variable de respuesta del rendimiento académico (RA) en función de las otras variables, se observa el efecto directo que incide con mayor fuerza es de la variable resentimiento $(\beta=-.411, p=.000)$ frente al resto de las variables exógenas como: agresividad $(\beta=-.002, p>.05)$, autonomía psicológica $(\beta=-.164$, $p<.001)$, control conductual, $(\beta=-.030, p>.05)$ y compromiso $(\beta=-.113, p<0.5)$, de los cuales representa mayor significancia estadística es el estilo autonomía psicológica y compromiso ( ver Tabla 2).

\section{Efecto Indirecto}

Se consideró como variable mediadora al resentimiento y se analizó los efectos indirectos, obteniendo resultados como: (1) En la asociación entre conducta agresiva y rendimiento académico, se obtuvo un efecto indirecto de $10.6 \%(-.106=.26 * .-41)$ frente al efecto directo $(\beta=.00)$; (2) Con respecto a la asociación entre el estilo de crianza autonomía psicológica y rendimiento académico se contrastó un efecto indirecto e inverso de $7.3 \%(-.073=-.18 * .-41)$ frente a un tamaño de efecto directo de $\beta=.16$ 
Tabla 2. Efecto directo e indirecto

\begin{tabular}{|c|c|c|c|c|c|}
\hline & & & \multirow{2}{*}{$\frac{\text { Indirecto }}{\beta_{1}}$} & \multicolumn{2}{|l|}{ Directo } \\
\hline & & & & $\beta_{2}$ & $P$ \\
\hline Autonomía Psicológica & $\rightarrow$ & Conducta agresiva & -.417 & & $* * *$ \\
\hline Control Conductual & $\rightarrow$ & Conducta agresiva & .350 & & $* * *$ \\
\hline Autonomía Psicológica & $\rightarrow$ & Resentimiento & -.179 & & $* * *$ \\
\hline Compromiso & $\rightarrow$ & Resentimiento & .111 & & .022 \\
\hline Conducta agresiva & $\rightarrow$ & Resentimiento & .268 & & $* * *$ \\
\hline Control Conductual & $\rightarrow$ & Resentimiento & -.149 & & .002 \\
\hline Compromiso & $\rightarrow$ & Rendimiento académico & & .113 & .011 \\
\hline Autonomía psicológica & $\rightarrow$ & Rendimiento académico & & .164 & $* * *$ \\
\hline Control Conductual & $\rightarrow$ & Rendimiento académico & & .030 & .498 \\
\hline Resentimiento & $\rightarrow$ & Rendimiento académico & & -.411 & $* * *$ \\
\hline Conducta agresiva & $\rightarrow$ & Rendimiento académico & & .002 & .968 \\
\hline
\end{tabular}

$(p<.000)$, (3). En el estilo de crianza control conductual y su asociación con el rendimiento académico se ha evidenciado un efecto indirecto y positivo de $6.1 \% \quad\left(.061=-.15^{*} \quad-.41\right)$ en comparación con un efecto $(\beta=.03, p>.05)$ y por último el estilo de crianza compromiso y su asociación con el rendimiento académico se ha observado un efecto indirecto y positivo de $4.5 \%$ $(.045=.11 *-.41)$.

\section{Discusión}

A partir del análisis de los resultados y considerando el objetivo principal del estudio de establecer un modelo explicativo del rendimiento académico en función a estilos de crianza, conducta agresiva y resentimiento, se puede decir que el efecto indirecto evidencia mayor implicancia en comparación con el directo. Lo que significa que los resultados mostraron un mayor efecto indirecto, donde se observa coeficientes de determinación con significancia estadística promedio en la mayoría de los casos. (Ellis, 2010)

Así también se ha encontrado una relación inversa entre el estilo de crianza autonomía psicológica y el rendimiento académico, lo que significa que en la medida que los padres emplean estrategias democráticas, no represiva con sus hijos, estos pueden tener escaso éxito en su rendimiento académico. $\mathrm{Y}$ en cuanto a la relación con los estilos control conductual y compromiso, se ha encontrado una relación directa con el rendimiento académico, que de alguna manera refuerza el anterior hallazgo, en el sentido de que a mayor supervisión o control del comportamiento del adolescente y acercamiento emocional puede tener éxito o mejorar el rendimiento académico.
Estos resultados hacen notar que para el éxito escolar o un buen rendimiento académico no es suficiente dejar bajo la autonomía y responsabilidad de los estudiantes, sino que es necesario la supervisión, el control y acercamiento por parte de los padres, lo que concuerda con lo encontrado por Duchesne y Ratalle (2010) quienes indican que el estudiante es responsable de esforzarse en su aprendizaje; sin embargo, son los padres quienes deben fomentar esta responsabilidad, estar pendiente y animar a sus hijos para que cumplan sus metas educativas, desde una interacción gobernadas por las dimensiones de afecto y control. Es decir que el estilo de crianza puede ser una variable significativa para lograr el éxito o fracaso en el rendimiento académico.

Los resultados demuestran que el estilo de crianza autonomía psicológica tiene mayor incidencia explicativa sobre la conducta agresiva $(\beta=-.42, p<* * *)$, seguida por el estilo control conductual $(\beta=.35, \quad p<* * *)$ Al parecer estos resultados solo estarían explicando el $28 \%$ de la varianza de la conducta agresiva, siendo explicado el mayor porcentaje probablemente por condiciones y situaciones externas al núcleo familiar tal como ha sido reportada por otras investigaciones (Nieto, 2015; Rodríguez, 2015; Guevara, 2015; Aguiar \& Cuesta, 2012; Sánchez, 2010). Por otra parte, la variable resentimiento es explicado con mayor incidencia por la conducta agresiva $(\beta=.26, p<* * *)$ y en menor magnitud por el estilo compromiso $(\beta=.11, p<.05)$.

Otro de los resultados está referido a que las variables autonomía psicológica y control conductual estarían contribuyendo en los niveles de agresividad y resentimiento, respectivamente; lo que a su vez en alguna medida estarían 
afectando en los niveles de rendimiento académico. Se encontró también que más de la mitad de la muestra de estudio (57\%) tiene bajo rendimiento académico. Por otro lado, la relación entre la agresión y el resentimiento toma mayor interés debido a que están cumpliendo el rol mediador entre los estilos de crianza y rendimiento académico, asumiéndose que el control conductual y la autonomía psicológica como estilos de mayor incidencia están asociados con la agresión y el resentimiento.

En el análisis relacional de las variables no se ha evidenciado relación indirecta y significativa entre la agresividad y rendimiento académico, sin embargo, al realizar el análisis conjuntamente con la variable resentimiento como mediadora, se hace más fuerte e inversa esta relación de la conducta agresiva con el rendimiento académico. (Figura 1)

$\mathrm{Al}$ analizar las variables de estudio de manera estructural, el resentimiento toma el efecto de la agresión y se hace más fuerte al relacionarse con el rendimiento académico, encontrándose en la muestra de estudio un alto resentimiento (78\%), el cual puede manifestarse a través de emociones destructivas como la envidia o rencor sordo (Nietzsche, 2011) y sentimientos de maltrato y postergación, (León \& Romero, 1990). De estos hallazgos y planteamientos se puede establecer que las emociones se expresan también en conductas negativas aprendidas que inevitablemente van a influenciar en el contexto escolar y específicamente en el nivel de conocimientos que puede lograr el estudiante, a su vez puede tener consecuencias sobre su salud y bienestar emocional. Al respecto Scott y Mamadi (2016), haciendo referencia los estudios de Kinder y Sander (1996), plantean que todo sentimiento negativo se desarrolla temprano en la vida de una persona a través del aprendizaje social.

Estudios sobre el contexto familiar (Baeza, 2000; Salazar et al., 2009; Jorge \& Gonzales, 2017; Bonilla et al., 2017) reportan que el rol, los hábitos y actitudes de la familia influyen en el desempeño académico y en la forma como los estilos de crianza de los padres cumplen un rol importante en el desempeño académico, dando importancia al ambiente físico y social constituido por el hogar. Del mismo modo Carrión (2015) plantea que los padres son los principales gestores para el desarrollo de los hijos y son los pioneros en practicar los estilos de crianza que influyen en los procesos de socialización y aprendizaje. Hallazgos que son similares al presente estudio, en el sentido que los estilos de crianza afectan al rendimiento académico.

Finalmente, el modelo estructural del rendimiento académico relacionado con los estilos de crianza, la conducta agresiva y el resentimiento en adolescentes escolares, puede explicar el nivel de rendimiento académico, teniendo como predictor importante al resentimiento que toma la carga de la conducta agresiva. Estos hallazgos permiten visualizar el rendimiento académico desde la perspectiva de los estilos de crianza y su relación con la agresión y el resentimiento.

Los investigadores pueden hacer uso de los datos descritos en el presente estudio para ampliar la cobertura de muestras y correlacionar con otros factores.

\section{Conflicto de interés}

Los autores declaran no presentar ningún conflicto de interés.

\section{Referencias}

Adell, M. A. (2002). Estrategias para mejorar el desempeño académico de los adolescentes. Madrid: Pirámide.

Anabalón, M., Carrasco, S. Díaz, D., Gallardo C., \& Cárcamo, H. (2008). El compromiso familiar frente al desempeño académico de niños y niñas de educación general básica en la ciudad de Chillán. Horizontes Educacionales. 13 (1), 11-21. https://www.redalyc.org/articulo.oa?id=97912 446001

Ato, M., \& Vallejo, G. (2015). Diseños de investigación en Psicología. Madrid: Pirámide

Baeza S. (2000). El rol de la familia en la educación de los hijos. Publicación virtual de la Facultad de Psicología y Psicopedagogía de la USAL. I (3),1-10.

Bandalos, D. L., \& Finney, S. J. (2010). Factor analysis: Exploratory and confirmatory. En G. R. Hancock y R. O. Mueller (Eds.), Reviewer's guide to quantitative methods. Routledge: New York. 
Bandura, A. (1982) Teoría del aprendizaje social. Madrid: Espasa.

Baron, R. M., \& Kenny, D. A. (1986). "The moderator-mediator variable distinction in social psychological research: Conceptual, strategic, and statistical considerations". Journal of Personality and Social Psychology. 51(6), 1173-1182. Copyright by the American Psychological Association, Inc.00223514/86/S00.75

Bausela, E. (2018), PISA 2012: Ansiedad y bajo rendimiento en competencia matemática. Revista Iberoamericana de Diagnóstico y Evaluación - e Avaliação Psicológica, 1(46), 161-173. https://doi.org/10.21865

Beaducel, A., \& Herzberg, P. Y. (2006). On the performance of maximum likelihood versus means and variance adjusted weighted least squares estimation in CFA. Structural Equation Modeling: Multidisciplinary Journal. 2(13), 186-203. https://doi.org/10.1207/s15328007sem1302 2

Bonilla, C. E., Núñez, S. M., Domínguez, R., \& Callejas, J. E. (2017). Las relaciones intrafamiliares de apoyo como mecanismo explicativo de la conducta violenta en adolescentes escolarizados. Universitas Psychological. 16(4), 1-12. https://doi.org/10.11144/Javeriana.upsy164.riam

Buss A., \& Perry, M. (1992) The aggression Questionnaire. Journal of Personality and Social Psychology. 63(3), 452-459. https://doi.org/10.1037/0022-3514.63.3.452

Brown, T. A. (2006). Confirmatory factor analysis for applied research. New York: Guilford Press.

Carrión, F. M. (2015). Estilos de crianza en familias migrantes. (Tesis de Maestría). Universidad de Cuenca. Ecuador.

Craig, G., \& Baucum, D. (2001). Desarrollo Psicológico. Distrito Federal, México: Pearson Educación.

Dámaso, J. (1994) Agresión, resentimiento y autoestima en adolescentes de conducta antisocial. (Tesis de Licenciatura) De la base de datos Universidad San Martín de Porres. Lima, Perú.

Darling, N., \& Steinberg, L. (1993). Parenting style as context: An integrative model.
Psychological Bulletin. 113(3), 1-10. Copyright 1993 by the American Psychological Association, Inc.0033-2909/93

Del Barrio, V., Carrasco, M. A. Rodríguez, M. A., \& Gordillo, R. (2009). Prevención de la agresión en la infancia y la adolescencia. International Journal of Psychology and Psychological Therapy. 9(2), 101-107. http://www.infocop.es/view_article.asp?id=2316

Del Barrio, V., Holgado, P., \& Carrasco, M. A. (2012). Dimensionalidad de la empatía y su relación con depresión, agresión y conducta prosocial. Revista de Psicología y Educación. 7(2), 49-67. ISSN:1699-9517-e-ISSN:19899874

Domínguez, A, J., Álvarez R. E., \& Vásquez V. E. (2017) Dimensiones predictivas del constructo violencia escolar en la educación secundaria obligatoria. Revista de Investigación Educativas. $\quad 35(2), \quad 337-351$. https://doi.org/10.6018/rie.35.2.259471

Domínguez, G. J. (2012). Estilo de socialización parental y fracaso escolar en la ESO: Una nueva mirada (Tesis Doctoral). De la base de datos Tesis Doctorales en Red (TDR). http://hdl.handle.net/10803/8405

Dorsch, F. (1982) Diccionario de psicología. Barcelona. Ed. Herder. Traducción del Alemán.

Duchesne, S., \& Ratelle, C. (2010), "Parental behaviors and adolescents' achievement goals at the beginning of Middle Scholl: Emotional problems as potential mediators". Journal of Educational Psichology. 2(102), 497-507. doi:10.1037/a0019320

Ellis, P. D. (2010). The Essential Guide to Effect Sizes: Statistical Power, Meta-Analysis, and the Interpretation of Research Results. New York: Cambridge University Press.

Enríquez, C. L., Segura, A. M., \& Tovar, J. R. (2013), Factores de riesgo asociados a bajo rendimiento académico en escolares de Bogotá. Investigaciones Andina, 15(26), 653666.

http://www.redalyc.org/articulo.oa?id=239026 287004

Estévez, E., \& Jiménez, T. I. (2015). Conducta agresiva y ajuste personal y escolar en una muestra de estudiantes adolescentes 
españoles. Universitas Psychological, 14(1), 111-124.

doi:10.11144/Javeriana.upsy14-1.caap

Ferrando, P. J., \& Anguiano-Carrasco, C. (2010). El análisis factorial como técnica de investigación en psicología. Papeles del Psicólogo, 31(1), 18-33. http://www.cop.es/papeles

Gana, K., \& Broc, G. (2019). Structural Equation Modeling with Lavaan. New York: Wiley

Gibu Shimabukuro, Ricardo. (2016). Sobre el resentimiento y el perdón. Revista de Filosofía Open Insight, 7(12), 9-29. http://www.scielo.org.mx/scielo.php?script=sc i_arttext\&pid=S2007-2

Gil, M. (2015) Agresión y rendimiento académico. Universidad de Jaén, Psicologia. https://hdl.handle.net/10953.1/1914

González, D. (2002). El desempeño académico universitario: variables psicológicas. Sonora, México: UniSon.

González-Pienda, J., \& Núñez, J. C. (2005). La implicación de los padres y su incidencia en el rendimiento de los hijos. Revista de Psicología y Educación. 1(1), 115-134. ISSN 1699-9517

Guevara, J. (2015). Press Start, los videojuegos como recurso educativo: Una propuesta de trabajo con Minecraft y Ciencias Sociales. Revista Electrónica de Recursos en Internet sobre Geografía y Ciencias Sociales. https://www.raco.cat/index.php/Aracne/article /view/303736

Hu, L. T., \& Bentler, P. M. (1999). Cutoff criteria for fit indexes in covariance structure analysis: Conventional criteria versus new alternatives. Structural Equation Modeling: A Multidisciplinary Journal. 6(1), 1-55. doi.org/10.1080/10705519909540118

Izzedin-Bouquet, R., \& Pachajoa-Londoño, A. (2009). Pautas, prácticas y creencias acerca de crianza. Ayer y hoy. Liberabit. 15(2), 109115. http://www. scielo.org.pe/pdf

Jiménez, D. (2009). Estilos de crianza y su relación con el DA, análisis y propuestas de intervención. (Tesis de Maestría) De la base de datos UNAM. México.

Jorge, E., \& González, C. (2017). Estilos de crianza parental: Una revisión teórica.
Informes Psicológicos. 17(2), 39-66 doi.org/10.18566/infpsic.v17n2a02

Leiva, L., R. Rodrigo, Peña, F., Vargas, F., \& Scquicciarini, A. (2019), Detectando las dificultades emocionales y conductuales en la escuela: Validación de PSC-17. Revista Iberoamericana de Diagnóstico y Evaluación Evaluación - e Avaliação Psicológica. 50(1), 95-105. doi.org/10.21865/RIDEP50.1.08

León, R., \& Romero, C. (1990) Estudios acerca del resentimiento. Concejo Nacional de Ciencia y Tecnología (CONCYTEC) Lima. 1ra. Edición.

Lozano, A. (2003). Factores personales, familiares y académicos que afectan al fracaso académico en la Educación Secundaria. Revista Electrónica de Investigación Psicoeducativa y Psicopedagógica. 1(1), 4366.

http://www.redalyc.org/articulo.oa?id=293152 876002

Manzano S., López, S., Suárez S., \& Ruiz-Ariza, A. (2018) Análisis descriptivo y de relación entre la actividad física y el rendimiento académico en jóvenes estudiantes del centro de España. International Journal of Developmental and Educational Psychology. 1(2), 223-228. SSN: 0214-9877.

Mardia, K. (1970). Measures of multivariate skewness and kurtosis with applications. Biometrika. 57(3), 519-530. doi:org/10.1093/biomet/57.3.519

Ministerio de Educación (2017) El Perú en PISA 2015. Informe nacional de resultados. Lima, Perú

Morales, C. S., Félix, R. V., Rosas, P. M., López, C. F., \& Nieto, G. J. (2015) Prácticas de crianza asociadas al comportamiento negativista desafiante y de agresión infantil. Avances en Psicología Latinoamericana. 33(1), 57-76.

https://www.redalyc.org/articulo.oa?id=79933 768005

Muthén, B., \& Kaplan D. (1985). A comparison of some methodologies for the factor analysis of non-normal Likert variables. British Journal of Math-ematical and Statistical Psychology. 38, 171-189. doi.org/10.1111/j.2044-8317.1985.tb00832.x 
Nieto, M. E. (2015). La mejora del aprendizaje a través de las nuevas tecnologías y de la implantación del currículo bimodal. Multiárea. Revista de Didáctica. 7(2015), 730. https://doi.org/10.18239/mard.v0i7.692

Nietzsche's F. (2011) On the genealogy of morality: A critical guide. Edited by Simon May. Cambridge University Press.

Obregón, G. (2017) Resentimiento y agresividad en estudiantes de 5to de secundaria. Avances en Psicología: Revista de la Facultad de Psicología y Humanidades. UNIFE. 25(2) https://doi.org/10.33539/avpsicol.2017.v25n2. 355

Pérez, E., \& Medrano, L. (2010) Análisis factorial exploratorio: Bases conceptuales y metodológicas. Revista Argentina de Ciencias del Comportamiento. 2(1), 58-66. ISSN 18524206 www.psyche.unc.edu.ar/racc

Quintero, J. (2017) Características de las pautas de crianza y su relación con el rendimiento académico: Una revisión documental. Colecciones digitales. URI: http://hdl.handle.net/10656/5226

Ramírez, M. A. (2005). Padres y desarrollo de los hijos: Prácticas de crianza. Estudios Pedagógicos. 31(2), 167-177.

http://dx.doi.org/10.4067/S071807052005000200011

Rodríguez, D., \& Guzmán, R. (2019). Rendimiento académico de adolescentes declarados en situación de riesgo. Revista de Investigación Educativa. 37(1), 147-162. https://doi.org/10.6018/rie.37.1.303391

Rodríguez, M., \& Ruiz, M. (2008). Atenuación de la asimetría y de la curtosis de las puntuaciones observadas mediante transformaciones de variables: Incidencia sobre la estructura factorial. Psicológica: Revista de Metodología y Psicología Experimental. 29(2), 205-227. ISSN 02112159

Rosales-Sarabia, R., Rivera, S., Reidl, L., \& García, M. (2018) Perdón y resentimiento hacia la pareja: Desarrollo y validación de dos escalas. Revista Iberoamericana de Diagnóstico y Evaluación - e Avaliação Psicológica. 47(2), 141-156. doi.org/10.21865/RIDEP47.2.10
Sánchez Benítez, G. (2010). Las estrategias de aprendizaje a través del componente lúdico. http://marcoele.com/descargas/11/sanchezestrategiasludico.pdf

Scott Carter, J., \& Mamadi Corra (2016) Racial resentment and attitudes toward the use of force by police: An over-time trend analysis. Sociological Inquiry. 20(10) 1-20. https://doi.org/10.1111/soin.12136

Torío-López, S., Peña-Calvo, J. V., RodríguezMenéndez, M. C., Fernández García, C. M., Molina Martín, S., Hernández García, J., \& Inda Caro, M. M. (2013). Construir lo cotidiano: Programa de educación parental. Barcelona: Editorial Octaedro.

Vallejo, A., \& Mazadiego, T. (2006). Familia y rendimiento académico. Revista de Educación y Desarrollo - Universidad Veracruzana, 11. Julio-septiembre 